\title{
Comparison of Osteogenic Potential of Phenytoin with Dexamethasone in Cultured Dental Pulp Stem Cells
}

\author{
Mitra Asgharian-Rezaee ${ }^{1,2}$, Raheleh Alipour-Farmad ${ }^{1}$, Zahra Tayarani-Najaran*3
}

\begin{abstract}
Background: One of the adverse effects of phenytoin (diphenylhydantoin, DPH) is enlargement of facial features. Although there are some reports on anabolic action of phenytoin on bone cells, the osteogenic potential of DPH on mesenchymal stem cells has not been studied. The purpose of this study was to evaluate the osteogenic potential of DPH on dental pulp stem cells (DPSCs).

Methods: Human DPSCs were isolated and characterized by flow cytometry; presence of CD29 and CD44 and absence of CD34 and CD45 were performed to confirm the mesenchymal stem cells. Isolated DPSCs were differentiated either in conventional osteogenic medium with Dexamethasone or medium containing different concentration of phenytoin $(12.5,25,100$, and $200 \mu \mathrm{M})$. The osteogenic differentiation evaluated by performing western blot test for Runt-related transcription factor 2 (RUNX2), osteopontin and alkaline phosphatase (ALP) also alizarin red S staining to measure the mineralization of cells.

Results: Our results showed morphological changes and mineralization of DPSCs by using DPH were comparable with dexamethasone. Moreover, western blot results of DPH group showed significant increase of ALP, RUNX2 and osteopontin (OSP) in comparison with control.

Conclusions: The data of present study showed the osteogenic activity of phenytoin, considering as an alternative of dexamethasone for inducing osteogenic differentiation of dental pulp stem cells.
\end{abstract}

Keywords: Dental Pulp Stem Cells, Osteogenic Differentiation, Phenytoin.

\section{Introduction}

The multi-potency of dental pulp stem cells (DPSCs) of differentiation to adipocytes, myoblasts, neuron-like cells, chondrocytes and osteoblasts have made them a new tool for cell therapy. The DPSCs are attractive source for scientists being applied in tissue engineering and regenerative medicine (1). Stem cells from human dental pulp can effectively be differentiated to osteogenic cells in the media containing dexamethasone, ascorbic acid, and $\beta$ glycerophosphate (2). Since the advantages of using mesenchymal stem cell (MSCs) as promising tool for reconstructive medicine, replacing lost or damaged bone/dentin, there are research interest to improve extracellular matrix mineralization of MSCs (3-6).

Phenytoin (diphenylhydantoin, DPH) is an anticonvulsant medicine with bone mineralization side effects. The anabolic activity of DPH on bone has been shown by decreasing in alveolar bone loss in patients receiving DPH (7). Also proliferation of gingival fibroblasts and collagen synthesis as well as changes in homeostasis of calcium and metabolism of bone are reported in chronic use of phenytoin. The experimental studies have shown osteoblastic differentiation in culture of fetal rat calvaria cells (8), also bone formation and increase in bone volume in rat by 
using DPH $(9,10)$.

Since there is not any comparative study on osteogenesis capacity of DPH and conventional media, in the present study the effect of DPH on induction of osteogenic differentiation of human dental pulp stem cells (hDPSCs) was examined in compare with conventional media containing dexamethasone.

For this purpose, the osteogenic potency of DPH and dexamethasone have been compared in induction of mineralized nodules as well as the amount of alkaline phosphatase (ALP), Runtrelated transcription factor 2 (RUNX2) and osteopontin.

\section{Materials and methods' \\ Chemicals}

The $\alpha$-modified Eagle's medium ( $\alpha$-MEM) and Fetal bovine serum (FBS) from Gibco; antibodies against ALP, RUNX2 and Osteopontin from abcam (Cambridge, MA, USA); $\beta$-actin and anti-rabbit IgG from Cell Signaling technology (Boston, USA); ECL Western blotting detection reagent from Bio$\mathrm{RaD}$ (USA); protease inhibitor cocktail, phosphatase inhibitor cocktail, Triton X-100, phenylmethylsulfonyl fluoride and QuantiPro BCA Assay Kit were purchased from Sigma (Steinheim, Germany).

\section{Subjects}

The pulp samples of human third molar dentin were obtained from three healthy subjects (1828 years of age) who were visited for orthodontic or other dentistry purposes in dentistry clinic of Academic Center for Education, Culture and Research (ACECR), Mashhad, Iran. The protocol was approved by the Ethics Committee, Mashhad University of Medical Sciences and performed in accordance with the ethical standards of Declaration of Helsinki. Informed consent was signed by all donors.

\section{Isolation of stem cells}

Human DPSCs were isolated and characterized in the Cell Culture lab, School of Pharmacy, Mashhad University of Medical Sciences. Teeth were collected from people who signed informed consent. Briefly, the pulp contents of freshly extracted molar teeth were enzymatically digested using $3 \mathrm{mg} / \mathrm{ml}$ collagenase type I and $4 \mathrm{mg} / \mathrm{ml}$ dispase (SigmaAldrich, St. Louis, MO, USA) at $37{ }^{\circ} \mathrm{C}$ for 45 $60 \mathrm{~min}$. After centrifugation of the cell suspension at $500 \mathrm{~g}$ for five min, the cells were washed and maintained in $\alpha$-MEM supplemented with $20 \%$ fetal bovine serum (FBS) (Gibco-Invitrogen, Carlsbad, CA, USA), $100 \mathrm{U} / \mathrm{ml}$ penicillin, $100 \mu \mathrm{g} / \mathrm{ml}$ streptomycin, and $0.25 \mu \mathrm{g} / \mathrm{ml}$ amphotericin B (SigmaAldrich) at $37{ }^{\circ} \mathrm{C}$ in a humidified atmosphere with $5 \% \mathrm{CO}_{2}$ for three weeks. The medium was renewed every three days.

\section{Flow cytometry analysis}

Human DPSCs were subjected to flow cytometry to confirm the phenotypic characteristics of MSCs. After isolation and culture, stem cells were harvested on passage three and flow cytometry analysis was performed using a FACSCalibur (BD Biosciences, San Diego, USA). The data were analyzed with WinMDI software. The FITC labeled antibodies to human CD29, CD33, CD44, and CD45 (abcam, USA) were used to stain the DPSCs on passage 3-5.

\section{Cell culture}

Dental pulp stem cells (DPSCs) were cultured in following media, separately.

1- C: Basal cell culture media as described above and cells grown in this medium served as the control group.

2- OBM: Osteogenic basal medium was $\alpha$ MEM supplemented with $10 \%$ FBS, $1 \%$ PenStrep, $50 \mu \mathrm{g} / \mathrm{ml}$ L-ascorbic acid (Sigma, Germany) and $10 \mathrm{mM}$ glycerol phosphate disodium salt ( $\beta$-glycerophosphate).

3- ODM: The experimental groups consisted of cells grown in OBM with the addition of dexamethasone $(0.1 \mu \mathrm{M})$.

4- DPH: The experimental groups consisted of cells grown in OBM with the addition of DPH $(12.5,50,100$, and $200 \mu \mathrm{M})$.

The DPSCs were incubated in C, OBM, DPH, and ODM for 10 days, and the media were refreshed twice a week. 


\section{Osteogenic differentiation}

Cells at the fourth passages were cultured on 12well plates and divided into four groups:

Group 1: Cells maintained in the regular culture medium ( $\mathrm{C}$ medium) served as controls.

Group 2: Osteogenic differentiation was induced when cells reached $80-90 \%$ confluence, using osteo-induction medium (OBM medium) according to the protocol of (2) and composed of $\alpha$-MEM supplemented with $10 \%$ FBS, $1 \%$ PenStrep, $50 \mu \mathrm{g} / \mathrm{ml}$ L-ascorbic acid (Sigma, Germany), $10 \mathrm{mM}$ glycerol phosphate disodium salt ( $\beta$-glycerophosphate).

Group 3: cells incubated in osteogenic media containing $0.1 \mu \mathrm{M}$ Dexamethasone (ODM medium).

Group 4: The effect of DPH (in $\alpha$-MEM supplemented with $10 \%$ FBS, $1 \%$ Pen-Strep, 50 $\mu \mathrm{g} / \mathrm{ml} \quad$ L-ascorbic acid, $10 \quad \mathrm{mM} \quad \beta$ glycerophosphate) on osteoblastic differentiation of hDPSC cells in culture at concentrations of $12.5,50,100$, and $200 \mu \mathrm{M}$ was investigated.

All cells in four groups incubated for 10 days. Media were replaced twice per week with freshly-prepared regular or osteogenic media as described above.

\section{Alizarin red $S$ (ARS) staining}

To evaluate calcium deposit in cells, on day 10, cultured cells were fixed with ice-cold $10 \%$ formalin for $30 \mathrm{~min}$. After washing with distilled water, the cells on four groups were stained with $2 \%$ Alizarin Red S at room temperature in dark for $45 \mathrm{~min}$. The stained cells were washed twice with distilled water. Intracellular calcium deposition was monitored by light microscopy.

\section{Western Blot}

Cells were plated on $25 \mathrm{~cm}^{2}$ at the density of $1 \times$ $10^{6}$ cells $/ 25 \mathrm{~cm}^{2}$ flask and allowed to attach and grow to up to $80 \%$ confluence. After 10 days, whole cell lysates were obtained using hypotonic buffer (50 mM Tris-HCl, pH 7.4), $150 \mathrm{mM} \mathrm{NaCl}$, $1 \%$ triton $\mathrm{X}-100,1 \mathrm{mM}$ EDTA, $0.2 \%$ SDS, $1 \%$ protease inhibitor cocktail, $1 \%$ phosphatase inhibitor cocktail and $1 \mathrm{mM}$ phenylmethylsulfonyl fluoride, $1 \mathrm{mM} \mathrm{Na3SO} 4$ ). After centrifugation the clear lysates were immediately boiled in SDS sample buffer. After
SDS and polyacrylamide gel electrophoresis the protein extracts were transferred to PVDF membranes and exposed to the rabbit Antiosteopontin antibody (ab91655); rabbit anti-ALP (ab354), rabbit Anti-RUNX2 antibody (ab23981) diluted 1:2000 (abcam ${ }^{\circledR}$, Cambridge, MA, USA), anti- rabbit IgG, HRP linked antibody, as secondary antibodies (Cell Signaling Technology, MA, USA) diluted in TBS-T pH 7.6 (1:2000). The membranes were read by enhanced chemiluminescence using the ECL Western blotting detection reagent. Images were quantified using the Gel-Pro Analyzer v.6.0 Gel Analysis Software. Anti $\beta$-actin antibody was used as control of protein loading. Data were then normalized to values of background and of control actin band.

\section{Statistical analysis}

Data was analyzed using GraphPad Prism (version 5.0; USA). Data are presented as mean \pm SEM of three independent tests. One-way analysis of variance (ANOVA) was used to compare the mean values of outcome variables across the categorical variables (groups), followed by a post-hoc Dunnett's test for pairwise comparisons. Values of $p<0.05$ were considered to indicate statistical significance.

\section{Results}

\section{Cell morphology}

After enzymatic digestion cells were grown in $12.5 \mathrm{~cm}^{2}$ culture flasks. On day 21 they were reached $70-80 \%$ confluence (Fig. 1A). To confirm the minimal criteria of DPSCs as MSCs, cells from passages 3-5 of DPSC cultures were characterized with flow cytometry. While isolated DPSC exhibited CD29 and CD44 but they were negative for $\mathrm{CD} 34$ and $\mathrm{CD} 45$ which all representative of MSC nature. Following culture of DPSCs in osteogenic media, the deposition of calcium was observed in most cells within the culture (Fig. 1B).

\section{Extracellular calcium deposition by ARS assay}

After isolation, DPSCs are incubated in four different media for 10 days. On day 10 calcium deposition was examined using alizarin red $S$ staining, calcium deposition was observed in both 


\section{Asgharian-Rezaee M et al}

DPSCs received dexamethasone and different concentrations of DPH. However, no mineralized nodules were seen in the control group cultured in $\alpha$-MEM (Fig. 2).

A)

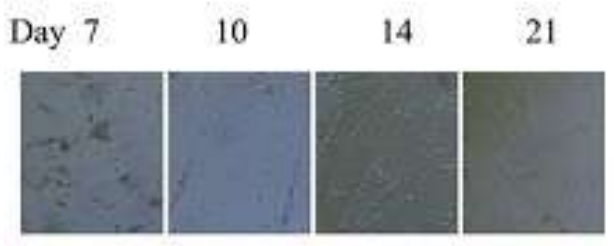

B)

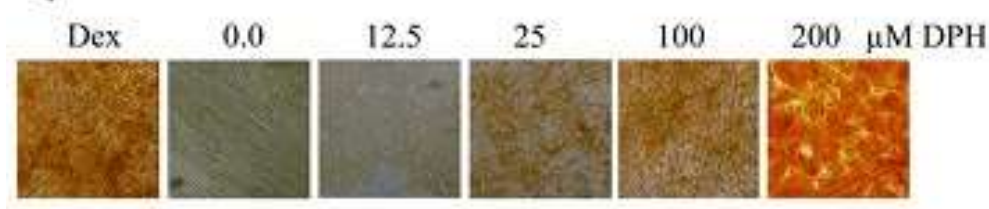

Fig. 1. (A): Cultured isolated DPSCs in $\alpha$-MEM medium on the day of $7,10,14,21$. (B): DPSCs cultured in conventional osteogenic media and DPH $(0-200 \mu \mathrm{M})$.
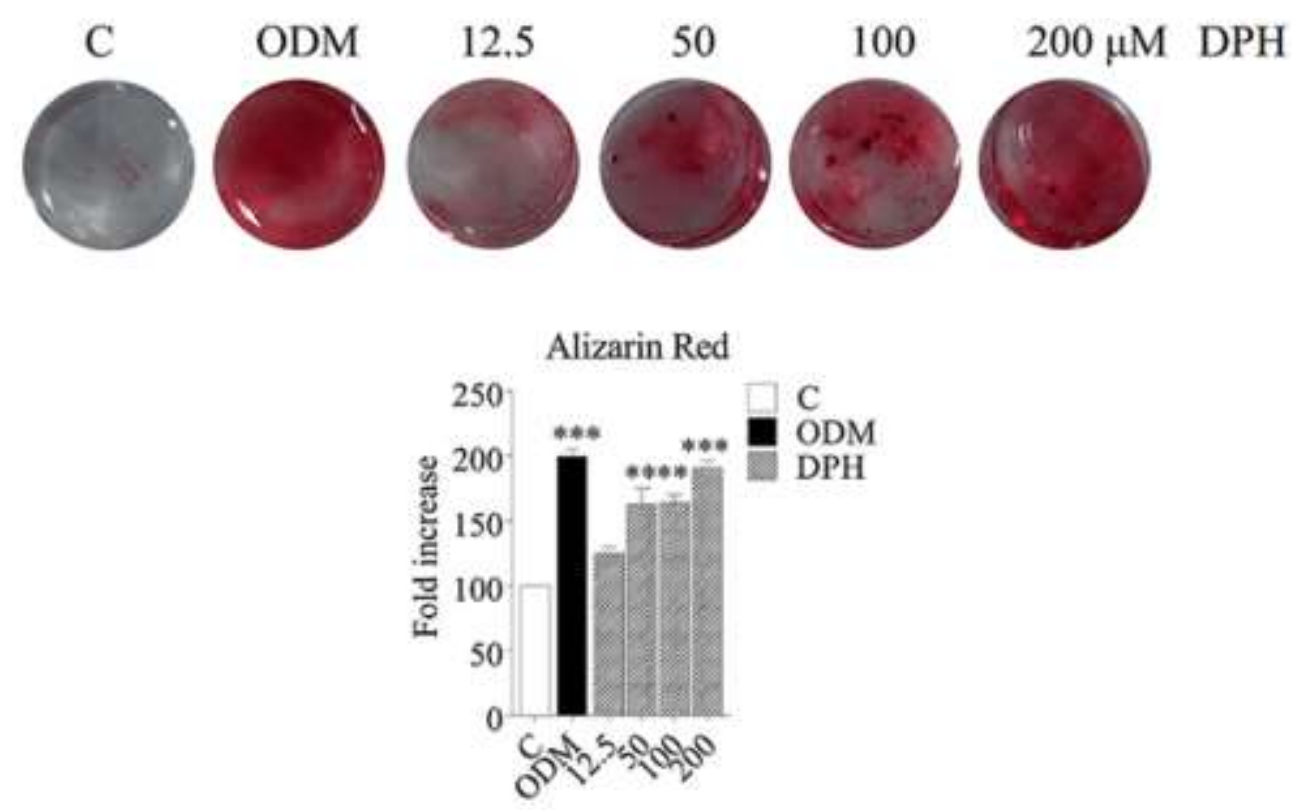

Concentration $(\mu \mathrm{M})$

Fig. 2. Alizarin red staining of hDPSCs cultured with conventional osteogenic media and DPH $(0-200 \mu \mathrm{M})$. Data are expressed as mean \pm SEM. $* \mathrm{p}<0.05, * * \mathrm{p}<0.01$ vs. control.

\section{Western blot analysis}

To confirm the osteogenic differentiation of hDPSCs in presence of DPH, specific osteoblast markers including osteopontin, RUNX2 and ALP were analyzed by western blot in the cells lysate of differentiated and control groups.

Once hydrolyzed by ALP, pyrophosphate can be used for bone mineralization as a source of phosphate (11). However, the generation pyrophosphate is under the influence of Runx2 transcription factor which is shown to promote the osteoblast differentiation of MSCs $(12,13)$. Also the formation and remodeling of bone is related to some other factors such as osteopontin that has an essential role in the tissue repair (14).

As our results, the expression levels of all examined proteins increased in DPH treated cells 
compared with control cells significantly $(\mathrm{p}<0.01$ for OS/ RUNX2 and p< 0.001 for ALP), (Fig. 3). There was no significant difference between expression of OSP and RUNX2 in DPH and dexamethasone groups, but the level of ALP was significantly higher in DPH group than dexamethasone especially in 100 and $200 \mu \mathrm{M}$ of $\mathrm{DPH}(\mathrm{p}<0.05)$.

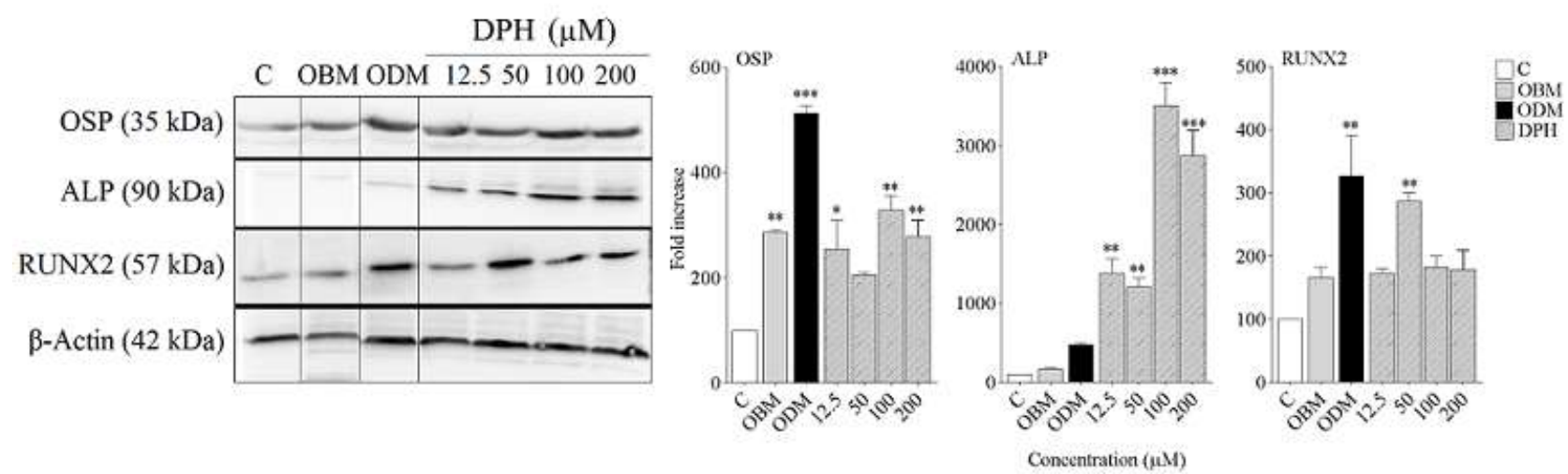

Fig. 3. Western blot analysis of Runt-related transcription factor 2 (RUNX2), osteopontin and alkaline phosphatase (ALP) DPHinduced osteogenic differentiation in DPSCs culture. Four groups were analyzed. Cells treated with: In 4 groups: C, OBM, ODM, DPH $(12.5,50,100$ and $200 \mu \mathrm{M})$ as described Methods and materials. The protein levels of alkaline phosphatase (ALP), Runtrelated transcription factor 2 (RUNX2) and osteopontin (OSP) were detected by western blot analysis at day 10 . $\beta$-Actin was used as the internal control. Data are expressed as mean \pm SEM. $* * p<0.01, * * * p<0.001$ vs. control.

\section{Discussion}

Recently dental pulp stem cells (DPSCs) are introduced as a source of mesenchymal stem cells which are widely used in basic and clinical researches. Upon stimulation under specific conditions, they have the potency of differentiation to adipocytes, neurons, myoblasts, chondrocytes and osteoblasts (1).

The anti-epileptic medicine; phenytoin (diphenylhydantoin, DPH) has been shown affecting the calcium homeostasis and/or bone metabolism. Although there are some reports on osteoblast formation in cell culture (8) but the effect of DPH on DPSCs has not been investigated yet. In spite of some studies on the ability of DPH to promote fatal stem cells to osteoblast there is not a comparative study between DPH and conventional supplement medium. Also the effect of DPH on osteogenic differentiation of MSCs is not clear. Here we tested the effect of DPH as an alternative of osteogenesis on MSCs. We also compared the DPH with conventional osteogenic medium contain dexamethasone, ascorbic acid, and $\beta$ glycerophosphate on DPSCs. This conventional medium has been widely used for differentiation of MSCs to osteoblasts $(15,16)$. It seems in this Supplement media dexamethasone has been implicated for transcriptional activation, ascorbic acid for collagen synthesis, and $\beta$ glycerophosphate for mineralization. There are some evidences for inhibitory effect of dexamethasone on growth of DPSCs (17), human MSCs (18) and human BMSCs (19) through cell-cycle arrest which has been contributed to osteogenic differentiation. Therefore, one of the main deals of finding the optimum environment for MSCs differentiation is investigation of formula and materials with osteogenic properties with minimal toxicity (20).

The results of our study showed DPH could increase markers related to transformation of DPSCs to osteoblasts including: ALP activity, RUNX2 and osteopontin in comparison with control.

DPH has been long used as an anticonvulsion agent which then introduced as a material that increases the osteoblast in tissues. The increase of osteoblast by DPH was considered when thickening of calvaria and coarsening of the facial features were reported in patients receiving DPH as the epilepsy treatment. The anabolic activity of DPH was the subject of some basic and clinical studies (9, 10, 21). However, in clinical studies, it has been shown 
that patients receiving DPH show less alveolar bone loss than untreated subjects (7). In an in vitro study osteogenosis has been shown in human-mandible-derived bone cells treated by DPH $(21,22)$. Similarly, DPH could increases the osteoblasts in human bone cells which reported to partly mediated by TGF-b (21). Also in other study in rats, DPH could stimulate the bone formation and increase the bone volume $(9,10)$.

Here we have shown that DPH could increase all osteogenic markers including RUNX2, ALP and osteopontin significantly compare with control. Moreover, ALP level was significantly higher in DPH treated cells in compare with dexamethasone group. However, mineralization in DPH treated group was comparable with dexamethasone specially in concentration of 100 and $200 \mu \mathrm{M}$ of DPH.

\section{References}

1. Ferro F, Spelat R, Baheney CS. Dental pulp stem cell (DPSC) isolation, characterization, and differentiation. Methods Mol Biol. 2014;1210:91115.

2. Spath L, Rotilio V, Alessandrini M, Gambara G, De Angelis L, Mancini M, et al. Explantderived human dental pulp stem cells enhance differentiation and proliferation potentials. J Cell Mol Med. 2010;14(6B):1635-44.

3. Mikami Y, Matsumoto T, Kano K, Toriumi T, Somei M, Honda MJ, et al. Current status of drug therapies for osteoporosis and the search for stem cells adapted for bone regenerative medicine. Anat Sci Int. 2014;89(1):1-10.

4. D'Alimonte I, Nargi E, Lannutti A, Marchisio M, Pierdomenico L, Costanzo G, et al. Adenosine A1 receptor stimulation enhances osteogenic differentiation of human dental pulp-derived mesenchymal stem cells via WNT signaling. Stem Cell Res. 2013;11(1):611-24.

5. Pozio A, Palmieri A, Girardi A, Cura F, Carinci F. Titanium nanotubes activate genes related to bone formation in vitro. Dent Res J (Isfahan). 2012;9(Suppl 2):S164-S168.

6. Huojia M, Muraoka N, Yoshizaki K, Fukumoto S, Nakashima M, Akamine A, et al. TGFbeta3 induces ectopic mineralization in fetal mouse
In conclusion our study showed osteogenic potential of DPH on DPSCs and may be considered as an alternative to conventional osteogenic medium. For better results, more investigation is needed to obtain optimum concentration of DPH for osteogenic differentiation in MSCs. As DPH is an old pharmacologic agent it could be safely used in regenerative medicine.

\section{Acknowledgements}

This study has been financially supported by the research council of Mashhad University of Medical Sciences and Kerman University of Medical Sciences. This paper is a part of MS thesis of the second author which has been registered as No. 931688 in research council of Mashhad University of Medical Sciences. The authors declare no potential conflicts of interest.

dental pulp during tooth germ development. Dev Growth Differ. 2005;47(3):141-52.

7. Seymour RA, Smith DG, Turnbull DN. The effects of phenytoin and sodium valproate on the periodontal health of adult epileptic patients. J Clin Periodontol. 1985;12(6):413-9.

8. Ikedo D, Ohishi K, Yamauchi N, Kataoka M, Kido J, Nagata T. Stimulatory effects of phenytoin on osteoblastic differentiation of fetal rat calvaria cells in culture. Bone. 1999;25(6):653-660.

9. Ohta T, Wergedal JE, Gruber HE, Baylink DJ, Lau KH. Low dose phenytoin is an osteogenic agent in the rat. Calcified Tissue International. 1995;56:42-48.

10. Ohta T, Wergedal JE, Matsuyama T, Baylink DJ, Lau KH. Phenytoin and fluoride act in concert to stimulate bone formation and to increase bone volume in adult male rats. Calcified Tissue International. 1995;56:390-397.

11. Hatch NE, Li Y, Franceschi RT. FGF2 stimulation of the pyrophosphate-generating enzyme, PC-1, in pre-osteoblast cells is mediated by RUNX2. J Bone Miner Res. 2009;24(4):652-662.

12. Komori T. Regulation of bone development and extracellular matrix protein genes by RUNX2. Cell Tissue Res. 2010;339(1):189-95. 
13. Sodek J, Ganss B, McKee MD. Osteopontin. Crit Rev Oral Biol Med. 2000;11(3):279-303.

14. Weng JJ, Su Y. Nuclear matrix-targeting of the osteogenic factor Runx2 is essential for its recognition and activation of the alkaline phosphatase gene. Biochim Biophys Acta. 2013;1830(3):2839-52.

15. Pisciotta A, Carnevale G, Meloni S, Riccio M, De Biasi S, Gibellini L, et al. Human dental pulp stem cells (hDPSCs): isolation, enrichment and comparative differentiation of two sub-populations. BMC Dev Biol. 2015;15:14.

16. Grottkau BE, Purudappa PP, Lin YF. Multilineage differentiation of dental pulp stem cells from green fluorescent protein transgenic mice. Int J Oral Sci. 2010;2(1):21-27.

17. Amir LR, Suniarti DF, Utami S, Abbas B. Chitosan as a potential osteogenic factor compared with dexamethasone in cultured macaque dental pulp stromal cells. Cell Tissue Res. 2014;358(2):407-15.

18. Wyles CC, Houdek MT, Wyles SP, Wagner ER, Behfar A, Sierra RJ. Differential cytotoxicity of corticosteroids on human mesenchymal stem cells. Clin Orthop Relat Res. 2015;473(3):1155-64. 19. Guzmán-Morales J, El-Gabalawy H, Pham MH, Tran-Khanh N, McKee MD, Wu W, et al. Effect of chitosan particles and dexamethasone on human bone marrow stromal cell osteogenesis and angiogenic factor secretion. Bone. 2009;45(4):617-626.

20. Monteiro N, Martins A, Ribeiro D, Faria S, Fonseca NA, Moreira JN, et al. On the use of dexamethasone-loaded liposomes to induce the osteogenic differentiation of human mesenchymal stem cells. J Tissue Eng Regen Med. 2015;9(9):1056-66.

21. Nakade O, Baylink DJ, Lau KH. Osteogenic actions of phenytoin in human bone cells are mediated in part by TGF-beta 1. J Bone Miner Res. 1996;11(12):1880-8.

22. Lau KH, Nakade O, Barr B, Taylor AK, Houchin K, Baylink DJ. Phenytoin increases markers of osteogenesis for the human species in vitro and in vivo. The Journal of Clinical Endocrinology \& Metabolism. 1995;80(8):23472353. 